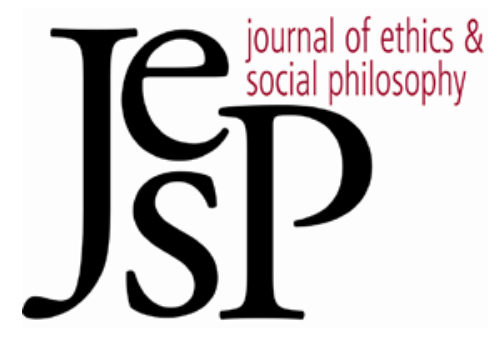

DISCUSSION NOTE

\title{
AgaINST SCANLON'S THEORY OF THE Strength of Practical Reasons
}

BY ERIC SAMPSON 


\title{
Against Scanlon's Theory of the Strength of Practical Reasons
}

\author{
Eric Sampson
}

\section{I}

F YOU ARE DYING OF STARVATION, you have stronger reason to eat something nourishing than to see to it that your desk is well stocked with paper clips. We could express the same truth by saying that your reasons to eat something nourishing outweigh, or are weightier than, your reasons to stock your desk with paper clips. But the notions of normative strength and weight ${ }^{1}$ are metaphors for some underlying normative reality. Any plausible theory of reasons and their strengths will need to cash out these metaphors. In Being Realistic about Reasons (2014), T. M. Scanlon offers a novel theory of normative strength. On Scanlon's view, a fact $p$ is a reason to perform an action or take some attitude $a$ when the relation $\mathrm{R}(p, x, c, a)$ holds between a fact, an agent, a circumstance, and an action or attitude. The strength of a reason is determined by facts about whether $p$ is a sufficient reason for $x$ to $a$ in either an actual or a counterfactual circumstance $c$. I argue that Scanlon's attempts to cash out the metaphor of normative strength by appealing to counterfactuals lead to deep problems for his view.

\section{Scanlon's Theory of Strength}

Some reasons outweigh others. Scanlon analyzes this outweighing relation in terms of the relation $\operatorname{SR}(p, x, c, a)$, which holds just in case a fact $p$ is a sufficient reason for $x$ to do $a$ in circumstances $c$. When the $\operatorname{SR}(p, x, c, a)$ relation holds, an agent who does $a$ in $c$ is not open to rational criticism for taking $p$ to be a sufficient reason to do a (Scanlon 2014: 106). Scanlon understands the outweighing relation as follows:

One consideration, $q$, outweighs another $p$, if the following hold: $\mathrm{R}(p, x, c, a), \mathrm{R}(q$, $x, c, b)$, where $b$ is a course of action incompatible with $a$, and $\operatorname{SR}(q, x, c, b)$ but not $\operatorname{SR}(p, x, c, a)$, although $\operatorname{SR}\left(p, x, c^{\prime}, a\right)$ where $c^{\prime}$ is a set of circumstances as normatively similar to $c$ as possible except that $q$ does not obtain in $c^{\prime}(108)$.

In other words, one reason $r$ outweighs another $r^{\prime}$ if $r$ is a sufficient reason to $\Phi$, but $r$ 'is not a sufficient reason to $\Psi$, where $\Phi$-ing and $\Psi$-ing are incompatible, but $r$ ' would have been a sufficient reason to $\Psi$ if the circumstances were normatively very similar but $r$ did not obtain.

Notice that Scanlon's characterization of the outweighing relation seems to rule out the possibility that there are facts about the relative weights of reasons for compatible actions. This is because the outweighing relation, as Scanlon sketches it, holds only between reasons for incompatible actions. Yet it

${ }^{1}$ I will use "strength" and "weight" interchangeably. 
seems clear that there are often facts about the relative strengths of reasons for different, but compatible, actions. Consider, for example, the strengths of my reasons in these two cases:

Bread Run: I am at the grocery store shopping for bread. There are two loaves left: $B_{1}$ and $B_{2}$. $B_{1}$ expires in three days. $B_{2}$ expires in seven days. $M y$ circumstances are such (let us say) that I have conclusive reason to grab $\mathrm{B}_{2}$.

Blue Button: If I do not push the blue button, everyone in Canada will die immediately. My circumstances are such that I have conclusive reason to push the blue button.

The actions described in these cases are compatible, since it is possible for an agent to grab $\mathrm{B}_{2}$ while in the grocery store and push the blue button when she is in a position to do so. Scanlon's account of outweighing seems to have nothing to say about the relative weights of my reasons in these cases. Nevertheless, it seems clear that the question, "Which reason is stronger: my reason to grab $B_{2}$ or my reason to push the blue button?" is both intelligible and has a determinate answer. My reason to push the blue button is (much) stronger.

But Scanlon has a reply. He can explain the relative weights of my reasons in Bread Run and Blue Button by imagining a counterfactual scenario in which I can either push the blue button or grab $\mathrm{B}_{2}$, but I cannot do both. In such a scenario, I would have sufficient reason to push the blue button, but I would not have sufficient reason to grab $\mathrm{B}_{2}$. On Scanlon's view, the truth of this counterfactual makes it the case that my reason to push the blue button is stronger than my reason to grab $\mathrm{B}_{2}{ }^{2}$

Scanlon's strategy for answering questions about the relative weights of reasons for two different but compatible actions, then, is to imagine counterfactual scenarios in which the two actions in question are incompatible. In other words, we should imagine scenarios in which the agent can act on one reason or the other, but not both. We then ask: On which reason does the agent have sufficient reason to act? The correct answer to this question determines which reason is stronger. Such a strategy seems to deliver the correct verdict when it comes to the relative strengths of my reasons in Bread Run and Blue Button. And Scanlon expects that similar appeals to counterfactuals will account for most of our intuitive judgments about the relative strengths of reasons across a wide range of other cases.

2 Though Scanlon does not consider this exact difficulty in his book, he has suggested to me in conversation that this is how he would like to account for strength comparisons between two conclusive reasons for compatible actions. And one can see that this is a natural extension of the view he puts forward in the book. 


\section{Against Scanlon's Theory of Strength}

Despite Scanlon's optimism, this strategy is bound to fail. There will be many cases in which there are clear facts about the relative strengths of two reasons $p$ and $q$, but it is impossible for $p$ and $q$ both to obtain in the same counterfactual scenario. Here is just one example. If I break my leg, I have a reason to get to a hospital immediately. If I step on a land mine and both of my legs are blown off, I have a reason to get to a hospital immediately. But I could not possibly be in a scenario in which I both have a broken leg and have no legs. If Scanlon's theory of strength is true, then there should be no fact of the matter about which of my reasons is stronger: my reason to get to the hospital when my I break my leg or my reason to get to the hospital when my legs are blown off. ${ }^{3}$ But, intuitively, there is a fact of the matter about which reason is stronger. I have (much) stronger reason to get to the hospital after I step on the land mine.

Scanlon might suggest that, in order to compare the strengths of reasons for compatible actions, we need not consider a single counterfactual scenario in which both reasons obtain. Rather, we could imagine two separate scenarios where one reason to $\Phi$ obtains in one world and the other reason to $\Phi$ obtains in the other world. We can then ask whether each reason is sufficient to $\Phi$ in their respective worlds. If one reason is sufficient to $\Phi$ and the other is not, then the former reason is stronger than the latter. For example, consider the question, "Which is stronger, my reason to get to a hospital after I break my leg or my reason to get to a hospital after my legs are blown off?" As I have argued, we cannot consider a single counterfactual scenario in which both reasons obtain - no such scenario is possible. But we can imagine two distinct, but very similar, scenarios: one in which I break my leg, and another in which my legs are blown off. And we can suppose further that, in each scenario, my mother is in desperate need of my help. She is in such need that my reason to get to a hospital immediately when I break my leg is not sufficient for me to get to a hospital immediately, but my reason to get to a hospital immediately when my legs are blown off is still sufficient to get to a hospital immediately. Scanlon could then appeal to the truth of these counterfactuals in order to explain why my reason to get to a hospital when I step on a land mine is stronger than my reason to get to a hospital when my leg is broken, even though it is impossible for both reasons to obtain in a single scenario. ${ }^{4}$

But this strategy will succeed only if the following principle is true:

3 Or perhaps Scanlon's view entails only that my reasons in each case are equally strong. Either way, such a result is problematic for Scanlon's theory of strength, since it seems clear that my reason to get to a hospital is much stronger when my legs are blown off than when my leg is broken.

4 Scanlon suggested precisely this reply to me in conversation. 
For any action $a$, and any two reasons to $a, p$ and $q$, such that it is impossible for $p$ and $q$ to obtain in the same circumstances, $p$ outweighs $q$ if the following hold: $\mathrm{R}(p$, $x, c, a), \mathrm{R}\left(q, x, c^{\prime}, a\right)$ and there is some counterfactual circumstance $c$ in which $\operatorname{SR}(p$, $x, c, a)$ but, in another counterfactual circumstance $c$ ' as normatively similar as possible to $c$, it is not the case that $\operatorname{SR}\left(q, x, c^{\prime}, a\right)$.

In other words, the principle Scanlon is relying on says that, if it is impossible for two reasons $r$ and $r$ ' to obtain in the same scenario, then if there is some scenario in which $r$ is a sufficient reason to $a$ but, in a scenario as normatively similar as possible, $r$ ' is not a sufficient reason to $a$, then $r$ is stronger than $r$ '. Unfortunately for Scanlon, this principle is false. It generates contradictions.

We have seen that, if the principle above is true, then my reason to get to a hospital when my legs are blown off is stronger than my reason to get to a hospital when I break my leg. That is because there is a counterfactual scenario in which my reason to get to a hospital when my legs are blown off is a sufficient reason to get to a hospital even though my mother needs help, but in the normatively similar situation in which I break my leg, I do not have a sufficient reason to get to a hospital immediately. We can, however, imagine two scenarios that are normatively very similar to one another in which the opposite is true. We could compare two scenarios - one in which I have a broken leg, one in which my legs are blown off - in which a mob of people on every road to every hospital is killing anyone who does not have two legs. ${ }^{5}$ In this set of counterfactual scenarios, the fact that I have a broken leg is a sufficient reason to get to a hospital, but the fact that my legs have been blown off is not. The verdict, then, on Scanlon's proposed strategy, will be that my reason to get to a hospital when my leg is broken is stronger than my reason to get to a hospital when my legs are blown off. If that is so, then we have a contradiction. The principle above, which Scanlon is relying on to defend his theory of strength, entails that my reason to get to a hospital when I have a broken leg is both stronger and not stronger than my reason to get to a hospital when my legs are blown off. That is because there is a scenario in which, according to the principle above, each reason is stronger than the other. So the principle above must be false. But if the principle above is false, then Scanlon's strategy for making strength comparisons between reasons that cannot obtain in the same scenario is doomed.

If what I have argued is correct, then there are facts about the relative strengths of my reasons to get to a hospital after I have sustained different injuries, but those facts are not determined by facts about counterfactual scenarios. And, if the facts about the relative weights of my reasons to get to a hospital are not determined by counterfactual scenarios, then there is good reason to think that the facts about the relative strengths of my reasons in situations with a similar structure - situations in which the reasons whose strengths are being compared could not possibly obtain in a single scenario are not determined by counterfactuals either. And, if my reasons in the range

${ }^{5}$ In these scenarios, my mother is not in need of assistance. 
of situations with that structure are not determined by facts about counterfactuals, then there is good reason to think that the relative strengths of reasons are never determined by counterfactuals. Otherwise, Scanlon would need to endorse a bizarre hybrid (or disjunctive) theory of strength: a theory according to which, in situations in which it is possible for the reasons being compared to obtain in the same scenario, the strengths of reasons are determined by counterfactuals and, when the reasons being compared cannot obtain in the same scenario, an entirely different theory of strength applies. But we should adopt this bizarre hybrid theory of strength only if all of the alternative theories of strength offering a unified (not disjunctive) account of strength (e.g., proportionalism, Schroeder's higher-order theory, the valuebased theory) fail terribly. 6 And we do not yet have reason to think that all of the other unified theories of strength fail in that way. Thus, we have good reason to conclude that the strengths of reasons must be determined, not by facts about counterfactuals, but by some other facts (e.g., facts about the relative strengths of agents' desires, facts about the relative values of certain states of affairs).

\section{Conclusion}

I have argued that any adequate theory of reasons and their strengths needs to be able to account for the relative strengths of reasons for different, but compatible, actions. Scanlon agrees and his proposal for accounting for these strength comparisons is to consider counterfactuals of various kinds. But this proposal has deep problems that are not easily remedied. There are many reasons that obviously have strengths relative to one another that could not possibly obtain in the same counterfactual scenario. If, in order to account for these strength comparisons, Scanlon tries to appeal to different but normatively similar counterfactual scenarios, then he will have to rely on a principle that generates contradictions. Perhaps Scanlon could adopt a hybrid theory of strength. But this hybrid theory would take a bizarre, ad hoc form. We should, therefore, look elsewhere for a theory of the strength of practical reasons. ${ }^{7}$

Eric Sampson

The University of North Carolina at Chapel Hill

Department of Philosophy

trae@live.unc.edu

${ }^{6}$ For defenses of these alternative unified theories of weight, see Manne (forthcoming), Schroeder (2007) and Maguire (unpublished).

7 I am grateful to the following people for helpful discussion about the ideas in this paper: Greg Nirshberg, David O’Brien, T. M. Scanlon, Russ Shafer-Landau, Shanna Slank and Mike Titelbaum. I owe special thanks to Alex Hyun for his critical and insightful comments on previous drafts of this paper. 


\section{References}

Maguire, B. (unpublished) "The Value-Based Theory of Reasons."

Manne, K. (forthcoming) "Democratizing Humeanism," in E. Lord and B. Maguire, eds., Weighing Reasons, Oxford: Oxford University Press.

Scanlon, T. M. (2014) Being Realistic about Reasons, New York: Oxford University Press.

Schroeder, M. (2007) Slaves of the Passions, New York: Oxford University Press. 